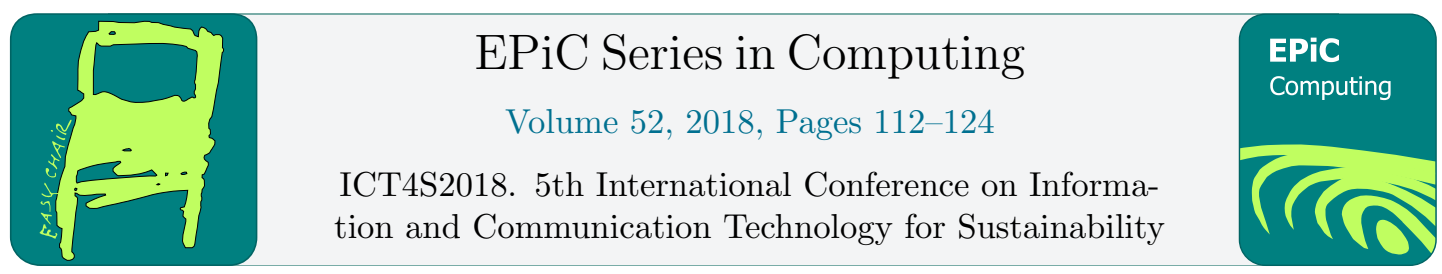

\title{
Indoor Temperature Awareness Using an Ambient Information Display - a Semi- longitudinal Study of one Household
}

\author{
Björn Hedin, Victor Larsson, Henrik Artman \\ KTH Royal Institute of Technology, Stockholm, Sweden \\ bjornh@kth.se, viclar@kth.se, artman@kth.se
}

\begin{abstract}
This paper explores the use of an Ambient Information Display (AID) to visualize indoor temperature, in order to promote behavior change through reflection and discussion. A prototype system was built using Philips Hue, a personal wireless LED lighting system, to visualize indoor temperature with colors, and an unused smartphone as temperature sensor. A household with a family of five was used as test environment. The design process underwent two major design iterations focusing on the visualization and its impact on the family's everyday perception of the indoor temperature, and the reflective processes this triggered. After three months of usage, late December to late March, the system was evaluated thoroughly. The awareness of the indoor temperature had been increased with the use of the system, where the AID had served as a trigger for discussions.
\end{abstract}

\section{Introduction}

Using persuasive technologies such as eco-visualization in order to promote energy conservation has been a popular research topic the last decade, and is a dominant topic in Sustainable HCI research (DiSalvo, Sengers, \& Brynjarsdóttir, 2010). Smart meters and visualizations have often used $\mathrm{kWh}$ as a main unit of feedback (Gamberini et al., 2012; Hargreaves, Nye, \& Burgess, 2010; McCalley, Kaiser, Midden, Keser, \& Teunissen, 2006; Murtagh et al., 2013; Yun et al., 2013) but this is a hard concept to relate to (Hedin \& Zapico, 2017; Karjalainen, 2011), and after an initial novelty effect the visualizations and smart meters are often more or less forgotten. An alternative to smart displays is to use ambient feedback where changes in light provides the feedback. Recent years has seen a dramatic increase in the availability of relatively low-cost lightbulbs (such as Philips Hue or more recently Ikea Trådlös) that can be programmatically controlled by easy to use interfaces such as IFTTT (IF This Then That, a free web-based service for connecting various other web services) combined with cheap sensors. In this paper we explore how such cheap consumer light systems can be used for eco 
feedback. We have chosen an intervention area with a high potential for energy conservation in many countries, indoor temperature. Potentially, such light-based feedback could be used for a number of other eco-feedback systems and it is therefore of interest to explore how such systems can be designed and how they are perceived by users.

Indoor temperature is an important topic for energy conservation in several countries. In 2015 in Sweden, $80.5 \mathrm{TWh}$ out of $375 \mathrm{TWh}$, or a bit more than $20 \%$ of the total energy use is used for heating (Swedish Energy Agency, 2015, 2016), and a normal house has to be heated during approximately 9 months of the year. Lowering temperature with one degree during these months decreases the amount of energy used with roughly $5 \%$, and lowering indoor temperature is the most important sustainability item on the top 10 list of energy saving suggestions by the Swedish Energy Agency. Lower indoor temperature also has health benefits (Fisk, 2000) and several studies suggest that indoor temperatures above $22-23^{\circ} \mathrm{C}$ increases Sick Building Syndrome symptoms (Fisk, 2000; Jaakkola, Heinonen, \& Seppänen, 1989). Therefore, individuals and families can have several reasons for wanting to reduce the temperature.

Several information campaigns have been launched informing the Swedish population about the benefits of lower indoor temperature. However, merely being aware of the positive effects of lowering indoor temperature rarely results in change (Michie, van Stralen, \& West, 2011). Several examples of systems that visualize energy consumption and temperature (e.g. a simple thermometer) have been available for a long time but rarely triggers any action (Gamberini et al., 2012; Hargreaves et al., 2010; Karjalainen, 2011; McCalley et al., 2006; Murtagh et al., 2013; Yun et al., 2013), or if it triggers action it is often a short-term change. In this paper we have chosen to investigate a long-term intervention instead.

We frame this study as design research, where we explore the use of Ambient Information Displays (AID) as a tool for a behavior change intervention. We use the Behavior Change Wheel framework (Michie et al., 2011) combined with the Behavior Change Taxonomy (Abraham \& Michie, 2008) to ground the design from a behavior change perspective, and we use Mankoff's principles for designing AIDs (Mankoff et al., 2003). The prototype system was developed iteratively, and evaluated semi-longitudinally.

The study does not aim at delivering statistically reliable data about how such a system works as a behavior change tool, but rather to explore how such a system could be designed, and to give guidance for further studies. Therefore, we have limited the study to include just one household for idea generation, requirement gathering and several evaluations during a $3+8$ month period.

\section{Theory and Related Research}

\subsection{Design of Behavior Change Interventions}

We have grounded the design in the Behavior Change Wheel framework, which is a relatively recent synthesis of several other behavior change frameworks, developed to help designing behavior change interventions (Michie et al., 2011). At its core (Figure 1) is the COM-B model which contains the three contextual components that should be present for behavior change interventions to work (Hedin, Lundström, Westlund, \& Markström, 2017). The "C" stands for the capability to perform the behavior. It can be either physical capability, such as having the physical strength to turn the knob on the radiator, or psychological capability, like having the knowledge how to adjust the indoor heat. The "O" stands for opportunity, and can be both physical opportunity like having access to the heating system, and social opportunity which can be whether it is socially acceptable or not to lower the heat. Finally, the "M" stands for motivation, which can be either automatic, i.e. making a behavior a habit 
like turning off a light bulb whenever you leave a room, or reflective motivation which involves reflective processes, like considering the consequences of actions and considering what is good and bad, acceptable or unacceptable.

The layer outside the Sources of behavior in the Behavior Change Wheel framework is intervention functions. These are the broad functions that behavior change interventions can aim at. The nine intervention functions are Education, Persuasion, Incentivisation, Coercion, Training, Restriction, Environmental restructuring, Modeling and Enablement.

The outer layer is seven policy categories, which we do not use in this paper.

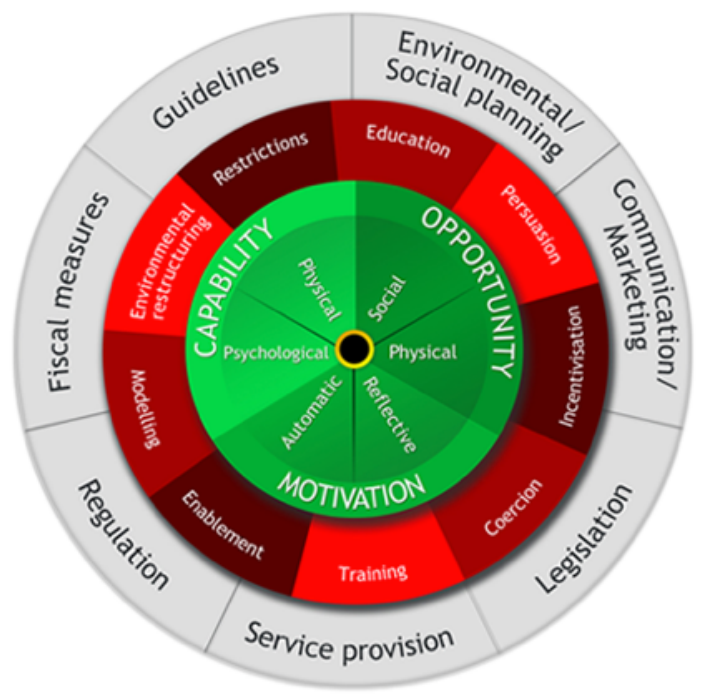

Figure 1: The Behavior Change Wheel

The actual Behavior Change Interventions implemented can then be mapped to the Behavior Change Taxonomy (BCT) (Michie et al., 2011). The BCT is a consensually agreed taxonomy of 93 behavior change interventions clustered into 16 groups. The 16 main groups are Goals and planning, Feedback and monitoring, Social support, Shaping knowledge, Natural consequences, Comparison of behavior, Associations, Repetition and substitution, Comparison of outcomes, Reward and threat, Regulations, Antecedents, Identity, Scheduled consequences, Self belief and finally Covert learning.

\subsection{ICT Design of Behavior Change Interventions for Sustainability using Ambient Light}

According to Brynjarsdottir (2012) few studies within persuasive sustainability have evaluated long-term effects, instead typically using a test period of 3-4 weeks. Most studies concern short studies with few subjects. The current study looks at one household but for a longer time. The argument is not to discuss sustainability as such but how a common system may impact the long-term awareness of indoor temperature for several persons within the household.

According to the behavior change wheel model, such feedback on behavior (temperature settings) can serve as an educational intervention in order to increase the psychological capability of acting differently (Michie et al., 2011).

According to Mankoff (2003), Ambient Information Displays (AID) can often be found as exhibitions or part of art found in museums, but from a human-computer interaction perspective, ambient displays have the goal of "presenting information without distracting or burdening the user" 
(ibid). A number of design principles were developed for ambient information displays that were taken into in this study consideration, quoted below: "

- Useful and relevant information: The information should be useful and relevant to the users in the intended setting.

- "Peripherally" of display: The display should be unobtrusive and remain so unless it requires the user's attention. User should be able to easily monitor the display.

- Match between design of ambient display and environments: One should notice an ambient display because of a change in the data it is presenting and not because its design clashes with its environment.

- Sufficient information design: The display should be designed to convey "just enough" information. Too much information cramps the display, and too little makes the display less useful.

- Consistent and intuitive mapping: Ambient displays should add minimal cognitive load. Cognitive load may be higher when users must remember what states or changes in the display mean. The display should be intuitive.

- Easy transition to more in-depth information: If the display offers multi-leveled information, the display should make it easy and quick for users to find out more detailed information.

- Visibility of state: An ambient display should make the states of the system noticeable. The transition from one state to another should be easily perceptible.

- Aesthetic and Pleasing Design: The display should be pleasing when it is placed in the intended setting."

Furthermore, semantic congruence is a powerful tool in order to create intuitive and informative displays as most people are able to detect the relation. For the purpose of visualizing temperature, color is very suitable since semantic congruence between temperature and color is strong (Ho, Van Doorn, Kawabe, Watanabe, \& Spence, 2014). Temperature and color has the congruence that red and blue indicate heat and cold respectively, which is present on virtually any device that has to do with temperature. Light has also the property of being conspicuous, as a bright light can cause a shift of attention (Goldstein, 2013). The author explains that to enhance the ability to catch attention an object should be easily distinguishable in the environment and lighter colors have higher salience, meaning that they draw more attention to them. Therefore, light together with colors may be a suitable feedback channel for applications where attention shift is needed, as it can catch the attention of the users. Furthermore, light has the possibility to emit a specific hue, brightness and saturation making it possible to explore the ability for visualization. Therefore in this study color was chosen to present temperature to the users in order to satisfy Mankoff's AID principle of "consistent and intuitive mapping".

Research about awareness of energy consumption with light as feedback has been done by Gustafsson \& Gyllenswärd (2005). The paper investigates how an ambient information system can be used to raise awareness to energy consumption by making electric cords emit light depending on how much energy that a particular cord is using. The authors describe how close the metaphor of light is to energy and how it can be used to display the amount of energy instead of a numeric display. A wizard-of-Oz experiment was conducted as evaluation of user feedback. The tested light programs were: static light with varied intensity, flowing light and pulsating light. The results show that static light was less informative but were perceived as calming in contrast to pulsing and flowing. Pulsating 
and flowing light were also considered intrusive. When the static intensity was high, some users thought it was uncomfortable.

Based on this we decided to base our system on static light, which satisfies Mankoff's design principle for "Peripherally of display". We further wanted to base the system on commercially available components that if possible could available even today in several homes. Based on this we set out to design a system.

\section{Behavior Design Rationale}

The Behavior Change Wheel framework has been used to identify the a) target behavior(s), b) source(s) of behavior that we wanted to affect, c) the intervention function(s) used, and d) to map the Behavior Change Techniques used to the Behavior Change Taxonomy.

The target behavior was decided to be the curtailment behavior to lower the indoor temperature when it is higher than necessary.

The sources of behavior were determined to be social opportunity (i.e. to support the user to lower the heat by making it socially acceptable by making it clearly visible to everyone that the current temperature is above a threshold), and reflective motivation (i.e. to make the users collectively reflect on their current practices regarding the objective measurement of indoor temperature and the subjective observation of feeling cold).

The intervention functions were Education and Persuasion.

Finally the Behavior Change Techniques use were mapped to the Behavior Change Taxonomy as follows (numbering from the Behavior Change Taxonomy):

2.4 Self-monitoring of outcomes of behavior. Everyone can clearly see whether the current temperature setting results in a cold, normal or high temperature.

3.3 Social support (emotional). Since the light makes the objective temperature visible to the entire family, a person can more easily bring up the topic of lowering the temperature.

7.1 Prompts/cues. The light prompts the user to consider lowering the temperature when the light turns red.

\section{Design and Iterations}

An initial brainstorm session among the authors of this paper resulted in a system design proposal where an old smartphone is connected to the local area network (LAN) and communicating with a Philips Hue LED light system for feedback on whether the household is below or over the average indoor temperature in the close area. The indoor temperature data should be fetched and stored in a web service, thus allowing for the number of users to easily scale. This became the basis for this project. The system can be divided into three main components based on the initial idea:

- setting up the old smartphone

- data collection

- visualization of data with ambient information display

The focus of this project was the visualization and data collection, as the application itself should have minimal interference. The flow for setting up the Philips Hue system is provided in the user manual for the system and is beyond the scope of this project. The flow, the steps and interfaces, for a user to connect an android application to the Hue system is provided in the startup project and will not be described either. To keep minimal interference with the system, the user should not interact with 
the smartphone after the setup, meaning that no specific user interface is needed after the setup. The overview of the system and its data flow is depicted in figure 2 .

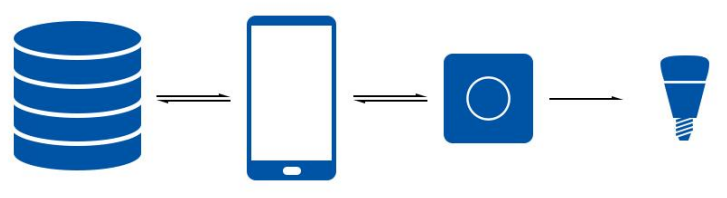

Figure 2: System overview. Smartphone communicates with web API and the Hue Bridge, which controls the light emitters.

\subsection{The Users in the Study}

A family offered their home as a testing environment. The house was built in the 1940's and has old insulation, meaning that the cost of heat is an interesting topic. Five people, four adults and one teenager are currently living in the house. The house has three floors and the temperature is regulated manually and heated by underfloor heating. The house is located north of Stockholm, Sweden. The family members are hereafter referred to as the users.

\subsection{Requirements}

The basic user requirements for the system had to be determined and extended from the initial idea combined with input from the users. A focus group session was conducted with the users for initial requirements. The focus group session was informal in its characteristics in order to allow for open brainstorming. A brief description of the project was done before discussing indoor temperatures and their thoughts about their indoor environment. The Hue light bulb was also presented. The session took about one and a half hour after the introduction, including a ten minute break.

The motivation from the users for using this kind of system was mainly to save energy and thereby reduce cost, and this was mainly a concern for the parents of the household. The system was highly welcomed as they thought it might be easier to perceive the temperature with visual feedback. An interesting and rather amusing case was presented by the parents when describing a recurring discussion in the family. The problem was in whether the adult children and the teenager was not wearing enough clothes to complain about too cold indoor temperature or if the temperature really was too low. A clear visible indicator of the temperature was agreed to be a solution as no thermometer was easy to access in the living room.

The indoor temperature was discussed and the users agreed that a scale of three is sufficient to provide useful information. The information the ambient light display should provide was whether the temperature is good (green), too cold (blue) or too hot (red). A discussion about how intensity or additional colors can visualize individual temperatures followed, but was discarded as it may be hard to perceive and remember, meaning that it should break the principle of low cognitive load by Mankoff (2003). One user was concerned about the light, arguing that it should be able to be customized to fit the style of the home. Another concern was the size of the Hue light bulb and that the light would affect the ambience of the room. The placement of the light source was suggested to be in the kitchen, as it was a common place to be in the home and is central in the house. 


\subsection{First Design Iteration}

According to the Public Health Agency of Sweden (Folkhälsomyndigheten, 2014), the recommended indoor temperature is 20-23 degrees Celsius. To motivate the household to lower their temperature, the conditions shown in Table 1 were set up. The hue values were taken from the chromaticity diagram provided by Philips Hue, so that blue light would mean cold, green light would mean ok, and red light would mean hot. The brightness and saturation of the colors were, as initial values, set to $100(0-254)$ in order to not be too vibrant or intense.

TABLE I. TEMPERATURE CHART FOR FIRST PROTOTYPE

\begin{tabular}{|l|l|}
\hline$t=$ temperature $[\mathbf{C}]$ & Color \\
\hline$t<20.25$ & Blue (hue $=25500$, brightness $=100$, saturnation $=100)$ \\
\hline $20.25<t<21.75$ & Green (hue $=46920$, brightness $=100$, saturnation $=100)$ \\
\hline$t>21.75$ & Red $($ hue $=65280$, brightness $=100$, saturnation $=100)$ \\
\hline
\end{tabular}

Based on the input from the focus group, the lamp, a bare Philips Hue light bulb, was deployed in the home in the kitchen near the dining room as shown in figure 2.

The first prototype was used without problems for four days with no data corruption. After interviewing the family as a group, the following responses were derived for each principle from Mankoff (2003):

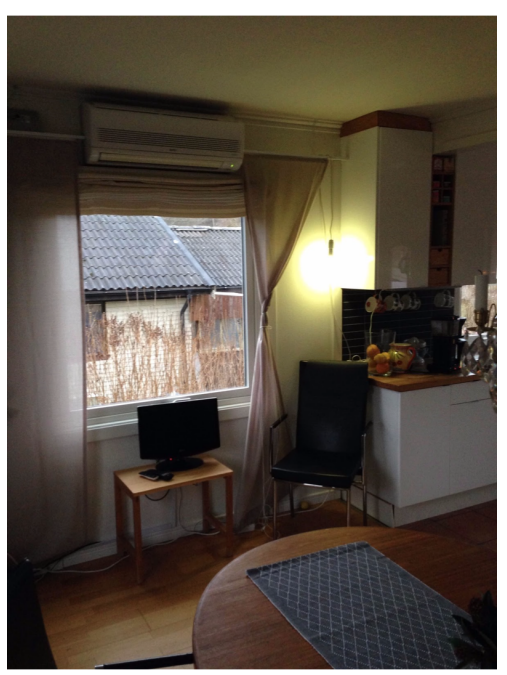

Figure 2: First prototype near the kitchen in the dining room

- Useful and relevant information: The display is useful and provided the intended feedback.

- "Peripherally" of display: The light was sometimes perceived as too bright, for example in the evening. Monitoring the display was easy, but the placement was asked to be changed as one cabinet blocked the view from the kitchen. 
- Match between design of ambient display and environments: The light was not aesthetically pleasing and did have affect the ambience when the environment were dark, e.g. in the evenings.

- Sufficient information design: The display showed the intended information.

- Consistent and intuitive mapping: The color mappings were easy to understand.

- Easy transition to more in-depth information: No in- depth information was available at the time, but the temperature visualization was of interest for the family.

- Visibility of state: The blue and green color was somewhat hard to distinguish when the environment was bright. The red color was easy to perceive.

- Aesthetic and Pleasing Design: The bare bulb was not really preferred and ideas about making the light as "modern art" was proposed in order to appear as a furniture.

The overall impression from the users was positive. They felt that their awareness was increased as they realized that the temperature was always at the red state in the evenings and during the night to be green or blue in the forenoon. As we had hoped, this visualization resulted in the family spontaneously discussing the difrerences. It seemed like a cold morning led to a manual raise in temperature and a decrease in temperature in the evening, creating a "yoyo" effect. A result of this was that they had thoughts about constraining the underfloor heating to only the ground and main floor, as the bedrooms were on the top floor.

As the temperature could be visualized with light over a great distance, a proposal to have a lamp and sensor in their motor home to monitor the temperature without having to leave the house, which is an interesting use case.

\subsection{Second Design Iteration}

The second design iteration was aimed towards improving the colors from the lamp based on the evaluation from the first design iteration. As there were problems distinguishing the blue from the green when the environment was bright, the smartphone now sends different color states to the Hue depending on the time of day as the days are drastically shorter in the winter in Sweden. The night/day threshold was based on the time for the sunrise and sunset in Stockholm.

To investigate the color, brightness and saturation, two short focus group sessions were set up, one in daylight and one at night, with the adults in the family to discuss new colors. The Hue was controlled by the application provided by Philips to control the Hue, which makes it easy to change the output color hue, brightness and saturation. Table 2 shows the resulting settings from the two sessions. 
TABLE II. SETTINGS FOR DIFFERENT COLORS

\begin{tabular}{|l|l|l|l|}
\hline \multirow{2}{*}{ Time of day } & \multicolumn{3}{|c|}{ Color } \\
\cline { 2 - 4 } & \multicolumn{1}{|c|}{ Blue } & \multicolumn{1}{c|}{ Green } & \multicolumn{1}{c|}{ Red } \\
\hline \multirow{3}{*}{ Day } & hue $=25500$ & hue $=46920$ & hue $=65280$ \\
& brightness $=50$ & brightness $=50$ & brightness $=50$ \\
& saturation $=254$ & saturation $=254$ & saturation $=254$ \\
\hline \multirow{3}{*}{ Night } & hue $=25500$ & hue $=46920$ & hue $=65280$ \\
& brightness $=10$ & brightness $=10$ & brightness $=10$ \\
& saturation $=254$ & saturation $=254$ & saturation $=254$ \\
\hline
\end{tabular}

\subsection{Formative Evaluation}

Improvement in the colors were clear as the blue and green became more distinguishable according to the users. The brightness was lowered significantly and made the emission more subtle. From an interview with the family after three weeks of usage, the following principles from Mankoff (2003) were changed:

- "Peripherally" of display: The visualization is now more distinguishable and can still be monitored easily.

- Match between design of ambient display and environments: As the brightness was lowered, less impact on the environment were perceived.

- Consistent and intuitive mapping: The colors can now be easily distinguished which makes the mapping clearer.

- Visibility of state: The colors were improved.

As the colors were hard to separate before, more than one principle were affected by updating the colors. The next design iteration was decided to be conducted after a number of months of usage in order to be able to evaluate the long term impact on the system.

\subsection{Formative Evaluation}

A workshop was conducted with the users to discuss the ability to gain insight from the AID. The AID was appreciated by the users as they directly gained insight about the roller-coaster like temperature the home had during the time. The users knew the house was old and the insulation in the second floor is poor, but they did not realize that the temperature was varying as much. The heating was described to be "very slow" and the reason of the peaks could be the "yoyo" effect created when increasing the heat when it is cold inside and decreasing when it is hot. As a result, an investment in better insulation was discussed among the users. There was also an interest in visualizing the outdoor temperature as it might affect the indoor temperature in an old house like the household the users lived in.

After evaluating the interface, the user suggested it would be interesting to also visualize the outdoor temperature. Another solution proposed was the visualization of temperature tendency. One additional light would indicate whether the temperature is rising or not. How that would be visualized was discussed without increasing the cognitive load. Pulsating light was proposed but according to Gustafsson \& Gyllenswärd (2005) pulsating light might be intrusive. No proposal was decided during the workshop. 


\section{Results after using the Prototype}

After nearly three months of usage, a focus group session was conducted to evaluate the system and its usage, and the learning effect from using it. The light was dismounted before the session and the session was conducted in the dining room from where the light had been clearly visible.

The users quickly agreed that light had been affecting their awareness of the indoor temperature, and had promoted both reflection and discussions. The main use they all agreed on was to validate their perception of the temperature, meaning that when they felt cold, a quick glance to the light would verify that it was objectively cold inside or whether they were just subjectively feeling cold. This led to the realization that they either had to put on some clothes or deal with that fact that they feel cold.

The focus group session concluded with them agreeing on positives and negatives about the system. The main positives were:

- increased awareness of the current indoor temperature resulting in the family members reflecting on their indoor temperature

- easy interaction

- initiated discussion about the indoor temperature, and specifically about improving insulation for the house

And the main negatives were:

- no exact temperature

- $\quad$ no temperature trends visualized

Finally, after 11 months ( 8 months after the end of the test period) a meeting with the family was set up one last time in November 2016 in order to see if the use of the prototype had had any longterm effects. The family said they had less knowledge about the current indoor temperature now than during the study since in order to see the temperature they now 1) had to find or go to a thermometer and 2) in case of the parents find their reading glasses in order to be able to read the small text on the thermometer. They also missed an easily available "objective" measurement of temperature, since the subjective feeling was very different if for example a person sitting still and studying and another person that has just finished a running session. During the test period the presence of the Hue light in the kitchen had created discussions about temperature, but with the light gone these discussion no longer occurred on a regular basis. The family had not yet done anything about the insulation since it required both a lot of planning and was a large investment, but they still planned to do it at some later date. However, after finishing the test period they had installed heavier drapes around the doors and windows in order to keep the heat inside the building and the temperature more even. They were still interested in a similar visualization of the outdoor temperature, "to visually see the temperature without having to find glasses would be nice".

\section{Discussion}

From a behavior change point of view, the system appeared to work mostly as intended. Reflective motivation was one of source of behavior that we tried to affect, where we intended our system to both make the participants more aware of the actual temperature and to sparc discussions about indoor temperature. All three behavior change techniques we used (self-monitoring of outcomes of behavior, 
emotional social support and prompts/cues) worked towards that goal. The other source of behavior was social opportunity, where our main intent was that the participants would feel more comfortable in lowering the heat when it was "objectively" too high. This worked to some extent, but also worked in the other way by making the participants put on a sweater if they felt cold but the lights showed that the feeling was subjective rather than objective. Other positive effects included to initiate a discussion about investing in better insulation, and that the family had invested in thicker drapes to prevent heat from escaping.

The use of ambient light as a simple and direct feedback for indoor temperature worked well after the initial tuning of the system. The application of Mankoff's principles as a guide to the design of the system was useful both as a checklist, and as criteria to evaluate the design against. For this system where only blue, green and red was used, the amount of cognitive load was low and therefore did not confuse the users. More temperatures could have been visualized and was something the participants would have liked, but this would have been harder to perceive. Temperature tendency was the other aspect the participants would have liked, but which was difficult to achieve with a one-lamp setup. If two or more laps had been used it would have been possible to visualize both current temperature and temperature trend, but that increase in information would also have meant a higher cognitive load. When discussing the simplicity of the current temperature visualization, the advantage of the quick overview and the use of light was clear.

Our conclusions are that Ambient Information Displays using a one lightbulb setup where colors were used to inform about indoor temperature worked relatively well, both to convey the information and to serve as non-intrusive information tool. The relatively long period the system was used, where the user kept the interest in the feedback given by the system, could indicate that this kind of feedback system is a way to go beyond the novelty effect of such systems that is a common criticism. However, even with the relatively simple case of visualizing temperature, the lack of detail (exact temperature, and temperature tendency) suggests that the possible use-cases for AIDs is relatively limited, unless Mankoff's principle "Easy transition to more in-depth information" can be solved.

\subsection{Limitations and Future Work}

This work has been mainly exploratory, and there are several limitations in the study, which future work could address. The primary aspect is of course that the study was only conducted on one family in one specific house. The solution could in future work be tested on more families and under more conditions such as different types of houses, with different heating systems, and also to take individual differences into account. This could result in both better reliability and validity, and deeper general insights. From a development point of view, our main suggestions for future work is to investigate how lightbulbs such as Philips Hue or Ikea Trådlös can be used to present more information without increasing the cognitive load, and how more in-depth information can be provided.

\section{References}

Abraham, C., \& Michie, S. (2008). A taxonomy of behavior change techniques used in interventions. Health Psychology, 27(3), 379.

Brynjarsdottir, H., Håkansson, M., Pierce, J., Baumer, E., DiSalvo, C., \& Sengers, P. (2012). Sustainably Unpersuaded: How Persuasion Narrows Our Vision of Sustainability. In Proceedings of the SIGCHI Conference on Human Factors in Computing Systems (pp. 947956). New York, NY, USA: ACM. http://doi.org/10.1145/2207676.2208539

DiSalvo, C., Sengers, P., \& Brynjarsdóttir, H. (2010). Mapping the Landscape of Sustainable HCI. In 
Proceedings of the SIGCHI Conference on Human Factors in Computing Systems (pp. 19751984). New York, NY, USA: ACM. http://doi.org/10.1145/1753326.1753625

Fisk, W. J. (2000). Health and productivity gains from better indoor environments and their relationship with building energy efficiency. Annual Review of Energy and the Environment, 25(1), 537-566.

Folkhälsomyndigheten. (2014). Folkhälsomyndighetens allmänna råd om temperatur inomhus Folkhälsomyndighetens författningssamling. Retrieved from https://www.folkhalsomyndigheten.se/globalassets/publicerat-material/foreskrifter1/fohmfs2014-17.pdf

Gamberini, L., Spagnolli, A., Corradi, N., Jacucci, G., Tusa, G., Mikkola, T., ... Hoggan, E. (2012). Tailoring feedback to users' actions in a persuasive game for household electricity conservation. In International Conference on Persuasive Technology (pp. 100-111). Springer.

Goldstein, E. B. (2013). Sensation and perception. Belmont, Calif. : Wadsworth ;

Gustafsson, A., \& Gyllenswärd, M. (2005). The power-aware cord: energy awareness through ambient information display. In CHI'05 extended abstracts on Human factors in computing systems (pp. 1423-1426). ACM.

Hargreaves, T., Nye, M., \& Burgess, J. (2010). Making energy visible: A qualitative field study of how householders interact with feedback from smart energy monitors. Energy Policy, 38(10), 6111-6119.

Hedin, B., Lundström, A., Westlund, M., \& Markström, E. (2017). The Energy Piggy Bank - A Serious Game for Energy Conservation. In The Fifth IFIP Conference on Sustainable Internet and ICT for Sustainability Funchal, Portugal - December 6-7, 2017.

Hedin, B., \& Zapico, J. (2017). Kilowh.at -- Increasing Energy Awareness Using an Interactive Energy Comparison Tool. In P. W. de Vries, H. Oinas-Kukkonen, L. Siemons, N. Beerlage-de Jong, \& L. van Gemert-Pijnen (Eds.), Persuasive Technology: Development and Implementation of Personalized Technologies to Change Attitudes and Behaviors: 12th International Conference, PERSUASIVE 2017, Amsterdam, The Netherlands, April 4--6, 2017, Proceedings (pp. 175-185). Cham: Springer International Publishing. http://doi.org/10.1007/978-3-319-55134-0_14

Ho, H.-N., Van Doorn, G. H., Kawabe, T., Watanabe, J., \& Spence, C. (2014). Colour-temperature correspondences: When reactions to thermal stimuli are influenced by colour. PloS One, 9(3), e91854.

Jaakkola, J. J. K., Heinonen, O. P., \& Seppänen, O. (1989). Sick building syndrome, sensation of dryness and thermal comfort in relation to room temperature in an office building: need for individual control of temperature. Environment International, 15(1), 163-168.

Karjalainen, S. (2011). Consumer preferences for feedback on household electricity consumption. Energy and Buildings, 43(2), 458-467.

Mankoff, J., Dey, A. K., Hsieh, G., Kientz, J., Lederer, S., \& Ames, M. (2003). Heuristic evaluation of ambient displays. In Proceedings of the SIGCHI conference on Human factors in computing systems (pp. 169-176). ACM.

McCalley, T., Kaiser, F., Midden, C., Keser, M., \& Teunissen, M. (2006). Persuasive appliances: goal priming and behavioral response to product-integrated energy feedback. In International Conference on Persuasive Technology (pp. 45-49). Springer.

Michie, S., van Stralen, M. M., \& West, R. (2011). The behaviour change wheel: A new method for characterising and designing behaviour change interventions. Implementation Science, 6(1), 42. http://doi.org/10.1186/1748-5908-6-42

Murtagh, N., Nati, M., Headley, W. R., Gatersleben, B., Gluhak, A., Imran, M. A., \& Uzzell, D. (2013). Individual energy use and feedback in an office setting: A field trial. Energy Policy, 62, $717-728$.

Swedish Energy Agency. (2015). Energiläget 2015. Retrieved from 
https://www.energimyndigheten.se/contentassets/50a0c7046ce54aa88e 0151796950ba0a/energil aget-2015_webb.pdf

Swedish Energy Agency. (2016). Summary of energy statistics for dwellings and non-residential premises for 2016.2 Retrieved from http://www.energimyndigheten.se/globalassets/statistik/bostader/energistatistik-for-smahusflerbostadshus-och-lokaler-2016.pdf

Yun, R., Lasternas, B., Aziz, A., Loftness, V., Scupelli, P., Rowe, A., ... Zhao, J. (2013). Toward the design of a dashboard to promote environmentally sustainable behavior among office workers. In International Conference on Persuasive Technology (pp. 246-252). Springer. 